\title{
Characterization of relationships between the domains of two linear matrix-valued functions with applications Yongge Tian
}

CBE, Shanghai Business School, Shanghai, China

\begin{abstract}
One of the typical forms of linear matrix expressions (linear matrix-valued functions) is given by $A+B_{1} X_{1} C_{1}+\cdots+B_{k} X_{k} C_{k}$, where $X_{1}, \ldots, X_{k}$ are independent variable matrices of appropriate sizes, which include almost all matrices with unknown entries as its special cases. The domain of the matrix expression is defined to be all possible values of the matrix expressions with respect to $X_{1}, \ldots, X_{k}$. I this article, we approach some problems on the relationships between the domains of two linear matrix expressions by means of the block matrix method (BMM), the matrix rank method (MRM), and the matrix equation method (MEM). As application, we discuss some topics on the relationships among general solutions of some linear matrix equations and their reduced equations.
\end{abstract}

Mathematics Subject Classifications (2000): 15A09; 15A24; $15 \mathrm{~A} 27$

Keywords: Linear matrix expression; domain; matrix equation; general solution; generalized inverse

\section{Introduction}

Throughout this article, we denote by $\mathbb{C}^{m \times n}$ the set of all $m \times n$ complex matrices; by $A^{*}, r(A)$, and $\mathscr{R}(A)$ the conjugate transpose, the rank, and the range (column space) of a matrix $A \in \mathbb{C}^{m \times n}$, respectively; by $I_{m}$ the identity matrix of order $m$; and $[A, B]$ be a row block matrix consisting of $A$ and $B$. A matrix $A \in \mathbb{C}^{m \times m}$ is said to be EP (or range Hermitian) if $\mathscr{R}\left(A^{*}\right)=\mathscr{R}(A)$ holds. We next introduce the definition and notation of generalized inverses of a matrix. The Moore-Penrose inverse of $A \in \mathbb{C}^{m \times n}$, denoted by $A^{\dagger}$, is the unique matrix $X \in \mathbb{C}^{n \times m}$ satisfying the four Penrose equations

$$
\text { (i) } A X A=A \text {, (ii) } X A X=X \text {, (iii) }(A X)^{*}=A X \text {, (iv) }(X A)^{*}=X A \text {. }
$$

A matrix $X$ is called an $\{i, \ldots, j\}$-generalized inverse of $A$, denoted by $A^{(i, \ldots, j)}$, if it satisfies the $i$ th, $\ldots, j$ th equations in 1.1). The collection of all $\{i, \ldots, j\}$-generalized inverses of $A$ is denoted by $\left\{\left(A^{(i, \ldots, j)}\right\}\right.$. There are all 15 types of $\{i, \ldots, j\}$-generalized inverses for a given matrix $A$ by definition, but people are mainly interested in the types that involve the first equation:

$$
A^{\dagger}, \quad A^{(1,3,4)}, \quad A^{(1,2,4)}, \quad A^{(1,2,3)}, \quad A^{(1,4)}, \quad A^{(1,3)}, \quad A^{(1,2)}, \quad A^{(1)},
$$

which are usually called the eight commonly-used types of generalized inverses of $A$ in the literature; see e.g., 44,5. 18. In addition, we also denote by $P_{A}=I_{m}-A A^{\dagger}$ and $Q_{A}=I_{n}-A^{\dagger} A$ the orthogonal projectors (Hermitian idempotent matrices) induced from $A$. The Kronecker product of any two matrices $A$ and $B$ is defined to be $A \otimes B=\left(a_{i j} B\right)$. The vectorization operator of a matrix $A=\left[\mathbf{a}_{1}, \ldots, \mathbf{a}_{n}\right]$ is defined to be $\operatorname{vec}(A)=\vec{A}=\left[\mathbf{a}_{1}^{\top}, \ldots, \mathbf{a}_{n}^{\top}\right]^{\top}$. A well-known property on the vec operator of a triple matrix product is $\overrightarrow{A X B}=\left(B^{\top} \otimes A\right) \vec{X}$; see e.g., 3,20 .

Linear matrix expressions that involve variable matrices arise in a variety of problems in pure and applied mathematics. In the present paper we pursue our study of a general linear matrix expressions of the form

$$
f\left(X_{1}, X_{2}, \ldots, X_{k}\right)=A+B_{1} X_{1} C_{1}+B_{2} X_{2} C_{2}+\cdots+B_{k} X_{k} C_{k},
$$

where $A \in \mathbb{C}^{m \times n}, B_{i} \in \mathbb{C}^{m \times p_{i}}$, and $C_{i} \in \mathbb{C}^{q_{i} \times n}$ are given, and $X_{i} \in \mathbb{C}^{p_{i} \times q_{i}}$ are variable matrices, $i=1,2, \ldots, k$. Eq. (1.3) is usually called a Linear Matrix-Valued Function (LMVF), while the collection of all possible matrix values of $(1.3)$, called the domain of (1.3), is denoted schematically by

$$
\mathcal{D}_{f}=\left\{f\left(X_{1}, X_{2}, \ldots, X_{k}\right) \mid X_{i} \in \mathbb{C}^{p_{i} \times q_{i}}, i=1,2, \ldots, k\right\} .
$$

Eq. (1.3) includes many kinds of well-known matrix expressions with variable entries as its special cases, such as, $A+B X, A+B X C, A+B X+Y C$, see e.g., 25, 27, as well as various partially specified matrices, such as, $\left[\begin{array}{cc}A & B \\ C & ?\end{array}\right],\left[\begin{array}{cc}A & ? \\ ? & D\end{array}\right],\left[\begin{array}{cc}A & ? \\ ? & ?\end{array}\right]$, etc, see e.g., $, 1,7,8,11,12$. . There are many natural modifications of considering the LMVFs in mathematics and applications. Here we mention a few:

E-mail: yongge.tian@gmail.com 
(I) The general solution of a consistent linear matrix equation $A X=B$ is $X=A^{-} B+\left(I-A^{-} A\right) U$, where $A^{-}$denotes a $g$-inverse of $A$ and the matrix $U$ is arbitrary; the general solution of a consistent linear matrix equation $A X B=C$ can be written as $X=A^{-} C B^{-}+\left(I-A^{-} A\right) U_{1}+U_{2}\left(I-B B^{-}\right)$, where $U_{1}$ and $U_{2}$ are two arbitrary matrices.

(II) The general expression of $g$-inverse $A^{-}$can be written as $A^{-}=A^{\dagger}+\left(I-A^{\dagger} A\right) U_{1}+U_{2}\left(I-A A^{\dagger}\right)$, where $A^{\dagger}$ is the Moore-Penrose inverse of $A$, and $U_{1}$ and $U_{2}$ are two arbitrary matrices.

(III) Consider a Gauss-Markov model $\left\{\mathbf{y}, X \beta, \sigma^{2} \Sigma\right\}$, where $\Sigma$ is a known symmetric nonnegative definite matrix and $\sigma^{2}$ is an unknown positive parameter. The general expression of the weighted leastsquares estimator (WLSE) of $\beta$ with respect to a given weight matrix $W$ can be written as $\tilde{\beta}=$ $\left(\left(X^{\prime} W X\right)^{\dagger} X^{\prime} W+\left[I_{m}-\left(X^{\prime} W X\right)^{\dagger}\left(X^{\prime} W X\right)\right] U\right) \mathbf{y}$, where $U$ is an arbitrary matrix.

(IV) The best linear unbiased estimator (BLUE) of $X \beta$ in $\mathscr{M}=\left\{\mathbf{y}, X \beta, \sigma^{2} \Sigma\right\}$ is $G \mathbf{y}$, where $G=$ $[X, 0]\left[X, \Sigma Q_{X}\right]^{\dagger}+U\left(I_{m}-\left[X, \Sigma Q_{X}\right]\left[X, \Sigma Q_{X}\right]^{\dagger}\right)$, in which, $U$ is an arbitrary matrix and $Q_{X}=I_{m}-X X^{\dagger}$.

All these matrices are in fact LMVFs that involve one or two variable matrices. In such cases, people wish to know properties of these matrix expressions, for example, uniqueness (invariance), maximum and minimum possible ranks, range inclusions, norms, etc. The results obtained can be used to describe and solve the original problems.

As is known to all, one of the fundamental tasks in algebra is to establish and describe various algebraic equalities for operations of elements in the algebra. Assume that two matrix-valued functions $f\left(X_{1}, X_{2}, \ldots, X_{k}\right)$ and $g\left(Y_{1}, Y_{2}, \ldots, X_{l}\right)$ of the same size are given, and one wish to know the connections between the two domains $\mathcal{D}_{f}$ and $\mathcal{D}_{g}$. In this situations, we may divide the work into the following four situations

$$
\mathcal{D}_{f} \cap \mathcal{D}_{g} \neq \emptyset, \quad \mathcal{D}_{f} \supseteq \mathcal{D}_{g}, \quad \mathcal{D}_{f} \subseteq \mathcal{D}_{g}, \quad \mathcal{D}_{f}=\mathcal{D}_{g}
$$

Here we mention some examples on relations between two linear matrix expressions:

(a) When do two solvable linear matrix equations $A_{1} X_{1} B_{1}=C_{1}$ and $A_{2} X_{2} B_{2}=C_{2}$, where $X_{1}$ and $X_{2}$ have the same size, have a common solution?

(b) When do the set inclusions $\left\{A^{-}\right\} \cap\left\{B^{-}\right\}=\emptyset,\left\{D_{1}-C_{1} A_{1}^{-} B_{1}\right\} \cap\left\{D_{2}-C_{2} A_{2}^{-} B_{2}\right\}=\emptyset$, and $\left\{A^{-}+\right.$ $\left.B^{-}\right\} \cap\left\{C^{-}\right\}=\emptyset$, as well as the set equalities $\left\{A^{-}\right\}=\left\{B^{-}\right\},\left\{D_{1}-C_{1} A_{1}^{-} B_{1}\right\}=\left\{D_{2}-C_{2} A_{2}^{-} B_{2}\right\}$, and $\left\{A^{-}+B^{-}\right\}=\left\{C^{-}\right\}$hold?

(c) When do OLSEs and BLUEs under a $\left\{\mathbf{y}, X \beta, \sigma^{2} \Sigma\right\}$ coincide? and when OLSEs and BLUEs under two competing linear regression models $\left\{\mathbf{y}, X_{1} \beta, \sigma_{1}^{2} \Sigma_{1}\right\}$ and $\left\{\mathbf{y}, X_{2} \beta, \sigma_{2}^{2} \Sigma_{2}\right\}$ coincide?

These facts show that algebraic features and performances of the matrix set in (1.4) are necessarily worth for investigation from both theoretical and applied points of view. In fact, a class of fundamental and meaningful problems that have been identified in the matrix calculus is the characterization of relationships between two given LMVFs under various assumptions. In view of the above facts, the present author intends to investigate the relationships between two domains $\mathcal{D}_{1}$ and $\mathcal{D}_{2}$ generated from some special cases of (1.3) using the matrix range and rank methodology. We also discuss the connections among general solutions of some linear matrix equations and their reduced linear matrix equations.

\section{Preliminaries}

Block matrix, rank of matrix, and matrix equation are basic concepts in linear algebra, while the block matrix method (BMM), the matrix rank method (MRM), and the matrix equation method (MEM) are three fundamental and traditional analytic methods that are widely used in matrix theory and applications because they give one the ability to construct and analyze various complicated matrix expressions and matrix equalities in a subtle and computationally tractable way.

We next present a group of well-known results on ranks of matrices and matrix equations that are described by way of generalized inverses, MRM and BMM, which we shall use to deal with various matrix expressions and matrix equalities. 
Lemma 2.1 ( [13]). Let $A \in \mathbb{C}^{m \times n}, B \in \mathbb{C}^{m \times k}$, and $C \in \mathbb{C}^{l \times n}$. Then

$$
\begin{aligned}
r[A, B] & =r(A)+r\left(P_{A} B\right)=r(B)+r\left(P_{B} A\right), \\
r\left[\begin{array}{l}
A \\
C
\end{array}\right] & =r(A)+r\left(C Q_{A}\right)=r(C)+r\left(A Q_{C}\right), \\
r\left[\begin{array}{cc}
A & B \\
C & 0
\end{array}\right] & =r(B)+r(C)+r\left(P_{B} A Q_{C}\right) .
\end{aligned}
$$

In particular, the following results hold.

(a) $r[A, B]=r(A) \Leftrightarrow \mathscr{R}(A) \supseteq \mathscr{R}(B) \Leftrightarrow A A^{\dagger} B=B \Leftrightarrow P_{A} B=0$.

(b) $r\left[\begin{array}{l}A \\ C\end{array}\right]=r(A) \Leftrightarrow \mathscr{R}\left(C^{*}\right) \subseteq \mathscr{R}\left(A^{*}\right) \Leftrightarrow C A^{\dagger} A=C \Leftrightarrow C Q_{A}=0$.

(c) $r\left[\begin{array}{ll}A & B \\ C & 0\end{array}\right]=r(B)+r(C) \Leftrightarrow P_{B} A Q_{C}=0$.

Lemma $2.2\left([26)\right.$. Let $A_{i} \in \mathbb{C}^{m \times n_{i}}$, and denote $\widehat{A}_{i}=\left[A_{1}, \ldots, A_{i-1}, A_{i+1}, \ldots, A_{k}\right], i=1,2, \ldots, k$. Then

$$
(k-1) r\left[A_{1}, A_{2}, \ldots, A_{k}\right]+\operatorname{dim}\left[\mathscr{R}\left(\widehat{A}_{1}\right) \cap \mathscr{R}\left(\widehat{A}_{2}\right) \cap \cdots \cap \mathscr{R}\left(\widehat{A}_{k}\right)\right]=r\left(\widehat{A}_{1}\right)+r\left(\widehat{A}_{2}\right)+\cdots+r\left(\widehat{A}_{k}\right) .
$$

In particular, the following three statements are equivalent:

(a) $r\left[A_{1}, A_{2}, \ldots, A_{k}\right]=r\left(A_{1}\right)+r\left(A_{2}\right)+\cdots+r\left(A_{k}\right)$.

(b) $(k-1) r\left[A_{1}, A_{2}, \ldots, A_{k}\right]=r\left(\widehat{A}_{1}\right)+r\left(\widehat{A}_{2}\right)+\cdots+r\left(\widehat{A}_{k}\right)$.

(c) $\mathscr{R}\left(\widehat{A}_{1}\right) \cap \mathscr{R}\left(\widehat{A}_{2}\right) \cap \cdots \cap \mathscr{R}\left(\widehat{A}_{k}\right)=\{0\}$.

We also use the following well-known results in the sequel.

Lemma 2.3 ( [19]). Let

$$
A X=B
$$

be a given linear matrix equation, where $A \in \mathbb{C}^{m \times n}$ and $B \in \mathbb{C}^{m \times p}$ are known matrices, and $X \in \mathbb{C}^{n \times p}$ is an unknown matrix. Then, the following statements are equivalent:

(i) Eq. 2.5 is consistent.

(ii) $\mathscr{R}(A) \supseteq \mathscr{R}(B)$.

(iii) $r[A, B]=r(A)$.

(iv) $A A^{\dagger} B=B$.

In this case, the general solution of the equation can be written in the parametric form

$$
X=A^{\dagger} B+Q_{A} U,
$$

where $U \in \mathbb{C}^{n \times p}$ is an arbitrary matrix. In particular, (2.5) holds for all matrices $X \in \mathbb{C}^{n \times p}$ if and only if both $A=0$ and $B=0$, or equivalently, $[A, B]=0$.

Lemma 2.4 ( [19]). Let

$$
A X B=C
$$

be a given linear matrix equation, where $A \in \mathbb{C}^{m \times n}, B \in \mathbb{C}^{p \times q}$, and $C \in \mathbb{C}^{m \times q}$ are given. Then, the following statements are equivalent:

(i) Eq. 2.7 is consistent.

(ii) Both $\mathscr{R}(A) \supseteq \mathscr{R}(C)$ and $\mathscr{R}\left(B^{*}\right) \supseteq \mathscr{R}\left(C^{*}\right)$. 
(iii) Both $r[A, C]=r(A)$ and $r\left[\begin{array}{l}B \\ C\end{array}\right]=r(B)$.

(iv) $A A^{\dagger} C B^{\dagger} B=C$.

In this case, the general solution of (2.7) can be written in the parametric form

$$
X=A^{\dagger} C B^{\dagger}+Q_{A} U+V P_{B},
$$

where $U, V \in \mathbb{C}^{n \times p}$ are arbitrary matrices. In particular, (2.7) holds for all matrices $X \in \mathbb{C}^{n \times p}$ if and only if

$$
\text { either }[A, C]=0 \text { or }\left[\begin{array}{l}
B \\
C
\end{array}\right]=0 \text {. }
$$

Lemma 2.5 ( [2]). The matrix equation

$$
A_{1} X_{1}+X_{2} B_{2}=C
$$

is consistent if and only if

$$
r\left[\begin{array}{cc}
C & A_{1} \\
B_{2} & 0
\end{array}\right]=r\left(A_{1}\right)+r\left(B_{2}\right),
$$

or equivalently,

$$
P_{A_{1}} C Q_{B_{2}}=0 .
$$

Eq. 2.10 holds for all matrices $X_{1}$ and $X_{2}$ if and only if one of the following four block matrix equalities holds

$$
\left[\begin{array}{cc}
C & A_{1} \\
B_{2} & 0
\end{array}\right]=0
$$

Lemma 2.6 ( 14]). The matrix equation

$$
A_{1} X_{1} B_{1}+A_{2} X_{2} B_{2}=C
$$

is consistent if and only if the following four conditions hold

$$
\begin{aligned}
& r\left[C, A_{1}, A_{2}\right]=r\left[A_{1}, A_{2}\right], \quad r\left[\begin{array}{cc}
C & A_{1} \\
B_{2} & 0
\end{array}\right]=r\left(A_{1}\right)+r\left(B_{2}\right), \\
& r\left[\begin{array}{cc}
C & A_{2} \\
B_{1} & 0
\end{array}\right]=r\left(A_{2}\right)+r\left(B_{1}\right), \quad r\left[\begin{array}{c}
C \\
B_{1} \\
B_{2}
\end{array}\right]=r\left[\begin{array}{l}
B_{1} \\
B_{2}
\end{array}\right],
\end{aligned}
$$

or equivalently,

$$
P_{A} C=0, \quad P_{A_{1}} C Q_{B_{2}}=0, \quad P_{A_{2}} C Q_{B_{1}}=0, \quad C Q_{B}=0,
$$

where $A=\left[A_{1}, A_{2}\right]$ and $B=\left[\begin{array}{l}B_{1} \\ B_{2}\end{array}\right]$.

Lemma 2.7 ( 10,22 ). Equation 2.14 holds for all matrices $X_{1}$ and $X_{2}$ if and only if one of the following four block matrix equalities holds

$$
\text { (i) }\left[C, A_{1}, A_{2}\right]=0, \quad \text { (ii) } \quad\left[\begin{array}{cc}
C & A_{1} \\
B_{2} & 0
\end{array}\right]=0, \quad \text { (iii) } \quad\left[\begin{array}{cc}
C & A_{2} \\
B_{1} & 0
\end{array}\right]=0, \quad \text { (iv) }\left[\begin{array}{c}
C \\
B_{1} \\
B_{2}
\end{array}\right]=0
$$

Lemma 2.8 ( 21] $)$. The matrix equation

$$
A_{1} X_{1}+X_{2} B_{2}+A_{3} X_{3} B_{3}+A_{4} X_{4} B_{4}=C
$$


is consistent iff the following four conditions hold

$$
\begin{aligned}
& r\left[\begin{array}{cccc}
C & A_{1} & A_{3} & A_{4} \\
B_{2} & 0 & 0 & 0
\end{array}\right]=r\left[A_{1}, A_{3}, A_{4}\right]+r\left(B_{2}\right), \\
& r\left[\begin{array}{ccc}
C & A_{1} & A_{3} \\
B_{2} & 0 & 0 \\
B_{4} & 0 & 0
\end{array}\right]=r\left[\begin{array}{l}
B_{2} \\
B_{4}
\end{array}\right]+r\left[A_{1}, A_{3}\right], \\
& r\left[\begin{array}{ccc}
C & A_{1} & A_{4} \\
B_{2} & 0 & 0 \\
B_{3} & 0 & 0
\end{array}\right]=r\left[\begin{array}{l}
B_{2} \\
B_{3}
\end{array}\right]+r\left[A_{1}, A_{4}\right], \\
& r\left[\begin{array}{cc}
C & A_{1} \\
B_{2} & 0 \\
B_{3} & 0 \\
B_{4} & 0
\end{array}\right]=r\left[\begin{array}{l}
B_{2} \\
B_{3} \\
B_{4}
\end{array}\right]+r\left(A_{1}\right) .
\end{aligned}
$$

\section{Some fundamental results on relationships between domains of two linear matrix-valued functions}

We start with two groups of known results on the relationships between two matrix sets generated from the two simplest cases in (1.3).

Lemma 3.1 ( [25]). Given two domains of LMVFs:

$$
\mathcal{D}_{1}=\left\{A_{1}+B_{1} X_{1} \mid X_{1} \in \mathbb{C}^{p_{1} \times n}\right\} \text { and } \mathcal{D}_{2}=\left\{A_{2}+B_{2} X_{2} \mid X_{2} \in \mathbb{C}^{p_{2} \times n}\right\},
$$

where $A_{1}, A_{2} \in \mathbb{C}^{m \times n}, B_{1} \in \mathbb{C}^{m \times p_{1}}$, and $B_{2} \in \mathbb{C}^{m \times p_{2}}$ are known matrices, and $X_{1} \in \mathbb{C}^{p_{1} \times n}$ and $X_{2} \in \mathbb{C}^{p_{2} \times n}$ are variable matrices, we have the following results:

(a) $\mathcal{D}_{1} \cap \mathcal{D}_{2} \neq \emptyset$, i.e., there exist $X_{1}$ and $X_{2}$ such that $A_{1}+B_{1} X_{1}=A_{2}+B_{2} X_{2}$ if and only if $\mathscr{R}\left(A_{1}-A_{2}\right) \subseteq$ $\mathscr{R}\left[B_{1}, B_{2}\right]$.

(b) $\mathcal{D}_{1} \subseteq \mathcal{D}_{2}$ if and only if $\mathscr{R}\left[A_{1}-A_{2}, B_{1}\right] \subseteq \mathscr{R}\left(B_{2}\right)$.

(c) $\mathcal{D}_{1}=\mathcal{D}_{2}$ if and only if $\mathscr{R}\left(A_{1}-A_{2}\right) \subseteq \mathscr{R}\left(B_{1}\right)=\mathscr{R}\left(B_{2}\right)$.

Lemma 3.2 ( [25]). Given two domains of LMVFs:

$$
\mathcal{D}_{1}=\left\{A_{1}+B_{1} X_{1} C_{1} \mid X_{1} \in \mathbb{C}^{p_{1} \times q_{1}}\right\} \text { and } \mathcal{D}_{2}=\left\{A_{2}+B_{2} X_{2} C_{2} \mid X_{2} \in \mathbb{C}^{p_{2} \times q_{2}}\right\},
$$

where $A_{i} \in \mathbb{C}^{m \times n}, B_{i} \in \mathbb{C}^{m \times p_{i}}$, and $C_{i} \in \mathbb{C}^{q_{i} \times n}$ are given, and $X_{i} \in \mathbb{C}^{p_{i} \times q_{i}}$ are variable matrices, $i=1,2$, we have the following results:

(a) $\mathcal{D}_{1} \cap \mathcal{D}_{2} \neq \emptyset$ if and only if the following four conditions hold

$$
\begin{array}{ll}
\mathscr{R}\left(A_{1}-A_{2}\right) \subseteq \mathscr{R}\left[B_{1}, B_{2}\right], & \mathscr{R}\left(A_{1}^{*}-A_{2}^{*}\right) \subseteq \mathscr{R}\left[C_{1}^{*}, C_{2}^{*}\right], \\
r\left[\begin{array}{cc}
A_{1}-A_{2} & B_{1} \\
C_{2} & 0
\end{array}\right]=r\left(B_{1}\right)+r\left(C_{2}\right), & r\left[\begin{array}{cc}
A_{1}-A_{2} & B_{2} \\
C_{1} & 0
\end{array}\right]=r\left(B_{2}\right)+r\left(C_{1}\right) .
\end{array}
$$

(b) $\mathcal{D}_{1} \subseteq \mathcal{D}_{2}$ if and only if one of the following three conditions holds

(i) $\mathscr{R}\left[A_{1}-A_{2}, B_{1}\right] \subseteq \mathscr{R}\left(B_{2}\right)$ and $\mathscr{R}\left[A_{1}^{*}-A_{2}^{*}, C_{1}^{*}\right] \subseteq \mathscr{R}\left(C_{2}^{*}\right)$.

(ii) $B_{1}=0, \mathscr{R}\left(A_{1}-A_{2}\right) \subseteq \mathscr{R}\left(B_{2}\right)$, and $\mathscr{R}\left(A_{1}^{*}-A_{2}^{*}\right) \subseteq \mathscr{R}\left(C_{2}^{*}\right)$.

(iii) $C_{1}=0, \mathscr{R}\left(A_{1}-A_{2}\right) \subseteq \mathscr{R}\left(B_{2}\right)$, and $\mathscr{R}\left(A_{1}^{*}-A_{2}^{*}\right) \subseteq \mathscr{R}\left(C_{2}^{*}\right)$.

(c) $\mathcal{D}_{1}=\mathcal{D}_{2}$ if and only if one of the following five conditions holds

(i) $\mathscr{R}\left(A_{1}-A_{2}\right) \subseteq \mathscr{R}\left(B_{1}\right)=\mathscr{R}\left(B_{2}\right)$ and $\mathscr{R}\left(A_{1}^{*}-A_{2}^{*}\right) \subseteq \mathscr{R}\left(C_{1}^{*}\right)=\mathscr{R}\left(C_{2}^{*}\right)$.

(ii) $A_{1}=A_{2}, B_{1}=0$, and $B_{2}=0$. 
(iii) $A_{1}=A_{2}, B_{1}=0$, and $C_{2}=0$.

(iv) $A_{1}=A_{2}, B_{2}=0$, and $C_{1}=0$.

(v) $A_{1}=A_{2}, C_{1}=0$, and $C_{2}=0$.

As an extension, we have the following result on relationships between domains of two general matrix-valued functions, which we shall use in the latter part of the article.

Theorem 3.3. Given two domains of LMVFs:

$$
\begin{aligned}
& \mathcal{D}_{1}=\left\{A_{1}+B_{1} X_{1}+Y_{1} C_{1} \mid X_{1} \in \mathbb{C}^{p_{1} \times n_{1}}, Y_{1} \in \mathbb{C}^{m_{1} \times q_{1}}\right\}, \\
& \mathcal{D}_{2}=\left\{A_{2}+B_{2} X_{2} C_{2}+D_{2} Y_{2} E_{2} \mid X_{2} \in \mathbb{C}^{s_{2} \times t_{2}}, Y_{2} \in \mathbb{C}^{u_{2} \times v_{2}}\right\} .
\end{aligned}
$$

where $A_{1} \in \mathbb{C}^{m \times n}, B_{1} \in \mathbb{C}^{m \times p_{1}}, C_{1} \in \mathbb{C}^{q_{1} \times n}, A_{2} \in \mathbb{C}^{m \times n}, B_{2} \in \mathbb{C}^{m \times s_{2}}, C_{2} \in \mathbb{C}^{t_{2} \times n}, D_{2} \in \mathbb{C}^{m \times u_{2}}$, and $E_{2} \in \mathbb{C}^{v_{2} \times n}$ are known matrices, we have the following results:

(a) $\mathcal{D}_{1} \cap \mathcal{D}_{2} \neq \emptyset$ if and only if the following four conditions hold

$$
\begin{aligned}
& r\left[\begin{array}{cccc}
A_{2}-A_{1} & B_{1} & B_{2} & D_{2} \\
C_{1} & 0 & 0 & 0
\end{array}\right]=r\left[B_{1}, B_{2}, D_{2}\right]+r\left(C_{1}\right), \\
& r\left[\begin{array}{ccc}
A_{2}-A_{1} & B_{1} & B_{2} \\
E_{2} & 0 & 0 \\
C_{1} & 0 & 0
\end{array}\right]=r\left[\begin{array}{l}
C_{1} \\
E_{2}
\end{array}\right]+r\left[B_{1}, B_{2}\right], \\
& r\left[\begin{array}{ccc}
A_{2}-A_{1} & B_{1} & D_{2} \\
C_{1} & 0 & 0 \\
C_{2} & 0 & 0
\end{array}\right]=r\left[\begin{array}{l}
C_{1} \\
C_{2}
\end{array}\right]+r\left[B_{1}, D_{2}\right], \\
& r\left[\begin{array}{cc}
A_{2}-A_{1} & B_{1} \\
C_{1} & 0 \\
C_{2} & 0 \\
E_{2} & 0
\end{array}\right]=r\left[\begin{array}{c}
C_{1} \\
C_{2} \\
E_{2}
\end{array}\right]+r\left(B_{1}\right) .
\end{aligned}
$$

(b) $\mathcal{D}_{1} \supseteq \mathcal{D}_{2}$ if and only if one of the following four conditions holds

$$
\begin{aligned}
& r\left[\begin{array}{cccc}
A_{2}-A_{1} & B_{1} & B_{2} & D_{2} \\
C_{1} & 0 & 0 & 0
\end{array}\right]=r\left(B_{1}\right)+r\left(C_{1}\right), \\
& r\left[\begin{array}{ccc}
A_{2}-A_{1} & B_{1} & B_{2} \\
E_{2} & 0 & 0 \\
C_{1} & 0 & 0
\end{array}\right]=r\left(B_{1}\right)+r\left(C_{1}\right), \\
& r\left[\begin{array}{ccc}
A_{2}-A_{1} & B_{1} & D_{2} \\
C_{1} & 0 & 0 \\
C_{2} & 0 & 0
\end{array}\right]=r\left(B_{1}\right)+r\left(C_{1}\right), \\
& r\left[\begin{array}{cc}
A_{2}-A_{1} & B_{1} \\
C_{1} & 0 \\
C_{2} & 0 \\
E_{2} & 0
\end{array}\right]=r\left(B_{1}\right)+r\left(C_{1}\right) .
\end{aligned}
$$


(c) $\mathcal{D}_{1} \subseteq \mathcal{D}_{2}$ if and only if one of the following four conditions holds

$$
\begin{aligned}
& \text { (i) } r\left[B_{2}, D_{2}\right]=m \text { or } r\left[\begin{array}{cccc}
A_{1}-A_{2} & B_{1} & B_{2} & D_{2} \\
C_{1} & 0 & 0 & 0
\end{array}\right]=r\left[B_{2}, D_{2}\right], \\
& \text { (ii) } r\left(B_{2}\right)=m \text { or } r\left(E_{2}\right)=n \text { or } r\left[\begin{array}{ccc}
A_{1}-A_{2} & B_{1} & B_{2} \\
C_{1} & 0 & 0 \\
E_{2} & 0 & 0
\end{array}\right]=r\left(B_{2}\right)+r\left(E_{2}\right), \\
& \text { (iii) } r\left(C_{2}\right)=n \text { or } r\left(D_{2}\right)=m \text { or } r\left[\begin{array}{ccc}
A_{1}-A_{2} & B_{1} & D_{2} \\
C_{1} & 0 & 0 \\
C_{2} & 0 & 0
\end{array}\right]=r\left(C_{2}\right)+r\left(D_{2}\right), \\
& \text { (iv) } r\left[\begin{array}{l}
C_{2} \\
E_{2}
\end{array}\right]=n \text { or } r\left[\begin{array}{cc}
A_{1}-A_{2} & B_{1} \\
C_{1} & 0 \\
C_{2} & 0 \\
E_{2} & 0
\end{array}\right]=r\left[\begin{array}{l}
C_{2} \\
E_{2}
\end{array}\right] .
\end{aligned}
$$

(d) $\mathcal{D}_{1}=\mathcal{D}_{2}$ if and only if both (b) and (c) hold.

Proof. The fact $\mathcal{D}_{1} \cap \mathcal{D}_{2} \neq \emptyset$ is obviously equivalent to $A_{1}+B_{1} X_{1}+Y_{1} C_{1}=A_{2}+B_{2} X_{2} C_{2}+D_{2} Y_{2} E_{2}$ for some $X_{1}, Y_{1}, X_{2}$, and $Y_{2}$. Rewrite it as

$$
B_{1} X_{1}+Y_{1} C_{1}-B_{2} X_{2} C_{2}-D_{2} Y_{2} E_{2}=A_{2}-A_{1}
$$

and applying Lemma 2.8 to 3.17 leads to Result (a).

By (2.10), 2.12), and (3.17), the fact $\mathcal{D}_{1} \supseteq \mathcal{D}_{2}$ holds iff

$$
P_{B_{1}}\left(A_{2}-A_{1}\right) Q_{C_{1}}+P_{B_{1}} B_{2} X_{2} C_{2} Q_{C_{1}}+P_{B_{1}} D_{2} Y_{2} E_{2} Q_{C_{1}}=0
$$

holds for all $X_{2}$ and $Y_{2}$. By Lemma 2.7. (3.18) holds for all $X_{2}$ and $Y_{2}$ iff

$$
\begin{aligned}
& {\left[P_{B_{1}}\left(A_{2}-A_{1}\right) Q_{C_{1}}, P_{B_{1}} B_{2}, P_{B_{1}} D_{2}\right]=0,} \\
& {\left[\begin{array}{cc}
P_{B_{1}}\left(A_{2}-A_{1}\right) Q_{C_{1}} & P_{B_{1}} B_{2} \\
E_{2} Q_{C_{1}} & 0
\end{array}\right]=0,} \\
& {\left[\begin{array}{cc}
P_{B_{1}}\left(A_{2}-A_{1}\right) Q_{C_{1}} & P_{B_{1}} D_{2} \\
C_{2} Q_{C_{1}}
\end{array}\right]=0,} \\
& {\left[\begin{array}{c}
P_{B_{1}}\left(A_{2}-A_{1}\right) Q_{C_{1}} \\
C_{2} Q_{C_{1}} \\
E_{2} Q_{C_{1}}
\end{array}\right]=0,}
\end{aligned}
$$

which, by Lemma 2.1(c), are equivalent to 3.9 - 3.12).

By (3.17) and Lemma 2.7, the fact $\mathcal{D}_{1} \subseteq \mathcal{D}_{2}$ holds iff one of the following four equations

$$
\begin{aligned}
& P_{G}\left(A_{1}-A_{2}\right)+P_{G} B_{1} X_{1}+P_{G} Y_{1} C_{1}=0, \\
& P_{B_{2}}\left(A_{1}-A_{2}\right) Q_{E_{2}}+P_{B_{2}} B_{1} X_{1} Q_{E_{2}}+P_{B_{2}} Y_{1} C_{1} Q_{E_{2}}=0, \\
& P_{D_{2}}\left(A_{1}-A_{2}\right) Q_{C_{2}}+P_{D_{2}} B_{1} X_{1} Q_{C_{2}}+P_{D_{2}} Y_{1} C_{1} Q_{C_{2}}=0, \\
& \left(A_{1}-A_{2}\right) Q_{H}+B_{1} X_{1} Q_{H}+Y_{1} C_{1} Q_{H}=0
\end{aligned}
$$

hold for all $X_{1}$ and $Y_{1}$. Further by Lemma 2.7, (3.23) holds for all $X_{1}$ and $Y_{1}$ iff one of the following two conditions holds

$$
P_{G}=0 \text { or } r\left[\begin{array}{cc}
P_{G}\left(A_{1}-A_{2}\right) & P_{G} B_{1} \\
C_{1} & 0
\end{array}\right]=0
$$

which are equivalent to

$$
r\left[B_{2}, D_{2}\right]=m \text { or } r\left[\begin{array}{cccc}
A_{1}-A_{2} & B_{1} & B_{2} & D_{2} \\
C_{1} & 0 & 0 & 0
\end{array}\right]=r\left[B_{2}, D_{2}\right] ;
$$

(3.24 holds for all $X_{1}$ and $Y_{1}$ iff one of the following three conditions holds

$$
P_{B_{2}}=0 \text { or } r\left[\begin{array}{cc}
P_{B_{2}}\left(A_{1}-A_{2}\right) Q_{E_{2}} & P_{B_{2}} B_{1} \\
C_{1} Q_{E_{2}} & 0
\end{array}\right]=0 \text { or } Q_{E_{2}}=0
$$


which are equivalent to

$$
r\left(B_{2}\right)=m \text { or } r\left[\begin{array}{ccc}
A_{1}-A_{2} & B_{1} & B_{2} \\
C_{1} & 0 & 0 \\
E_{2} & 0 & 0
\end{array}\right]=r\left(B_{2}\right)+r\left(E_{2}\right) \text { or } r\left(E_{2}\right)=n ;
$$

3.25 holds for all $X_{1}$ and $Y_{1}$ iff one of the following four conditions holds

$$
P_{D_{2}}=0 \text { or } r\left[\begin{array}{cc}
P_{D_{2}}\left(A_{1}-A_{2}\right) Q_{C_{2}} & P_{D_{2}} B_{1} \\
C_{1} Q_{C_{2}} & 0
\end{array}\right]=0 \text { or } Q_{C_{2}}=0
$$

which are equivalent to

$$
r\left(D_{2}\right)=m \text { or } r\left[\begin{array}{ccc}
A_{1}-A_{2} & B_{1} & D_{2} \\
C_{1} & 0 & 0 \\
C_{2} & 0 & 0
\end{array}\right]=r\left(C_{2}\right)+r\left(D_{2}\right) \text { or } r\left(C_{2}\right)=n ;
$$

3.26 holds for all $X_{1}$ and $Y_{1}$ iff one of the following four conditions holds

$$
\begin{gathered}
Q_{H}=0 \text { or } r\left[\begin{array}{cc}
\left(A_{1}-A_{2}\right) Q_{H} & B_{1} \\
C_{1} Q_{H} & 0
\end{array}\right]=0, \\
r\left[\begin{array}{c}
C_{2} \\
E_{2}
\end{array}\right]=n \text { or } r\left[\begin{array}{cc}
A_{1}-A_{2} & B_{1} \\
C_{1} & 0 \\
C_{2} & 0 \\
E_{2} & 0
\end{array}\right]=r\left[\begin{array}{l}
C_{2} \\
E_{2}
\end{array}\right] .
\end{gathered}
$$

Combining them leads to $3.13-3.16$.

The results in the above three lemmas can be used, as demonstrated below, to solve many concrete problems on the relationships between solutions of matrix equations, as well as relations between generalized inverses of matrices.

\section{Relationships between solutions of two fundamental linear matrix equations}

It is well known since Penrose [19] that general solutions of linear matrix equations can be represented certain linear matrix expressions composed with the given matrices in the matrix equations and their generalized inverses. In this situation, we can use the previous results to characterize various relationships between solutions of linear matrix equations. There are many linear matrix equations for which the general solution can explicitly be written as certain explicit linear matrix-valued functions as given in 4.1. In this section, we present a variety of results and facts on relationships between linear transformations of solutions of some fundamental linear matrix equations.

Theorem 4.1. Assume that the following two matrix equations

$$
A_{1} X_{1}=B_{1} \text { and } A_{2} X_{2}=B_{2}
$$

are consistent, respectively, where $A_{i} \in \mathbb{C}^{m_{i} \times n_{i}}$ and $B_{i} \in \mathbb{C}^{m_{i} \times p}$ are given, $i=1,2$. Also we denote by

$$
\mathcal{D}_{1}=\left\{S_{1} X_{1}+T_{1} \mid A_{1} X_{1}=B_{1}\right\} \text { and } \mathcal{D}_{2}=\left\{S_{2} X_{2}+T_{2} \mid A_{2} X_{2}=B_{2}\right\},
$$

the domains of two constrained LMVFs, where $S_{i} \in \mathbb{C}^{s \times n_{i}}$ and $T_{i} \in \mathbb{C}^{s \times p}$ are given, $i=1,2$. Then the following results hold.

(a) $\mathcal{D}_{1} \cap \mathcal{D}_{2} \neq \emptyset$ if and only if $r\left[\begin{array}{ccc}S_{1} & S_{2} & T_{1}-T_{2} \\ A_{1} & 0 & -B_{1} \\ 0 & A_{2} & B_{2}\end{array}\right]=r\left[\begin{array}{cc}S_{1} & S_{2} \\ A_{1} & 0 \\ 0 & A_{2}\end{array}\right]$.

(b) $\mathcal{D}_{1} \subseteq \mathcal{D}_{2}$ if and only if $r\left[\begin{array}{ccc}S_{1} & S_{2} & T_{1}-T_{2} \\ A_{1} & 0 & -B_{1} \\ 0 & A_{2} & B_{2}\end{array}\right]=r\left[\begin{array}{l}S_{2} \\ A_{2}\end{array}\right]+r\left(A_{1}\right)$. 
(c) $\mathcal{D}_{1}=\mathcal{D}_{2}$ if and only if $r\left[\begin{array}{ccc}S_{1} & S_{2} & T_{1}-T_{2} \\ A_{1} & 0 & -B_{1} \\ 0 & A_{2} & B_{2}\end{array}\right]=r\left[\begin{array}{l}S_{1} \\ A_{1}\end{array}\right]+r\left(A_{2}\right)=r\left[\begin{array}{l}S_{2} \\ A_{2}\end{array}\right]+r\left(A_{1}\right)$.

Proof. By Lemma 2.3, the general solutions of the two equations in (4.1) can be expressed as

$$
X_{1}=A_{1}^{\dagger} B_{1}+S_{1} Q_{A_{1}} U_{1}, \quad X_{2}=A_{2}^{\dagger} B_{2}+S_{2} Q_{A_{2}} U_{2},
$$

where $U_{1} \in \mathbb{C}^{n_{1} \times p}$ and $U_{2} \in \mathbb{C}^{n_{2} \times p}$ are arbitrary matrices. Then the two sets in 4.2 can be represented as

$$
\mathcal{D}_{1}=\left\{S_{1} A_{1}^{\dagger} B_{1}+S_{1} Q_{A_{1}} U_{1}+T_{1}\right\} \text { and } \mathcal{D}_{2}=\left\{S_{2} A_{2}^{\dagger} B_{2}+S_{2} Q_{A_{2}} U_{2}+T_{2}\right\} .
$$

Applying Lemma 3.1. (a) to 4.4, we obtain that $\mathcal{D}_{1} \cap \mathcal{D}_{2} \neq \emptyset$ if and only if

$$
r\left[S_{1} Q_{A_{1}}, S_{2} Q_{A_{2}}, S_{1} A_{1}^{\dagger} B_{1}-S_{2} A_{2}^{\dagger} B_{2}+T_{1}-T_{2}\right]=r\left[S_{1} Q_{A_{1}}, S_{2} Q_{A_{2}}\right],
$$

where by 2.2 ,

$$
\begin{aligned}
& r\left[S_{1} Q_{A_{1}}, S_{2} Q_{A_{2}}, S_{1} A_{1}^{\dagger} B_{1}-S_{2} A_{2}^{\dagger} B_{2}+T_{1}-T_{2}\right] \\
& =r\left[\begin{array}{ccc}
S_{1} & S_{2} & S_{1} A_{1}^{\dagger} B_{1}-S_{2} A_{2}^{\dagger} B_{2}+T_{1}-T_{2} \\
A_{1} & 0 & 0 \\
0 & A_{1} & 0
\end{array}\right]-r\left(A_{1}\right)-r\left(A_{2}\right) \\
& =r\left[\begin{array}{ccc}
S_{1} & S_{2} & T_{1}-T_{2} \\
A_{1} & 0 & -B_{1} \\
0 & A_{2} & B_{2}
\end{array}\right]-r\left(A_{1}\right)-r\left(A_{2}\right), \\
& r\left[S_{1} Q_{A_{1}}, S_{2} Q_{A_{2}}\right]=r\left[\begin{array}{cc}
S_{1} & S_{2} \\
A_{1} & 0 \\
0 & A_{2}
\end{array}\right]-r\left(A_{1}\right)-r\left(A_{2}\right) .
\end{aligned}
$$

Substituting (4.6) and (4.7) into 4.5 yields $r\left[\begin{array}{ccc}S_{1} & S_{2} & T_{1}-T_{2} \\ A_{1} & 0 & -B_{1} \\ 0 & A_{2} & B_{2}\end{array}\right]=r\left[\begin{array}{cc}S_{1} & S_{2} \\ A_{1} & 0 \\ 0 & A_{2}\end{array}\right]$, establishing (a).

Applying Lemma 3.1(b) to (4.5), we obtain that $\mathcal{D}_{1} \cap \mathcal{D}_{2} \neq \emptyset$ if and only if

$$
r\left[S_{1} Q_{A_{1}}, S_{2} Q_{A_{2}}, S_{1} A_{1}^{\dagger} B_{1}-S_{2} A_{2}^{\dagger} B_{2}+T_{1}-T_{2}\right]=r\left(S_{2} Q_{A_{2}}\right),
$$

where by $(2.2)$,

$$
r\left(S_{2} Q_{A_{2}}\right)=r\left[\begin{array}{l}
S_{2} \\
A_{2}
\end{array}\right]-r\left(A_{2}\right)
$$

Substituting (4.6) and (4.9) into 4.8 yields Result (b). By a similar approach, we obtain that $\mathcal{D}_{1} \supseteq \mathcal{D}_{2}$ if and only if $r\left[\begin{array}{ccc}S_{1} & S_{2} & T_{1}-T_{2} \\ A_{1} & 0 & -B_{1} \\ 0 & A_{2} & B_{2}\end{array}\right]=r\left[\begin{array}{l}S_{1} \\ A_{1}\end{array}\right]+r\left(A_{2}\right)$. Combining it with Result (b) leads to Result (c).

Corollary 4.2. Assume that $A_{1} X_{1}=B_{1}$ and $A_{2} X_{2}=B_{2}$ in (4.1) are consistent, respectively, and denote by

$$
\mathcal{D}_{1}=\left\{X_{1} \mid A_{1} X_{1}=B_{1}\right\} \text { and } \mathcal{D}_{2}=\left\{X_{2} \mid A_{2} X_{2}=B_{2}\right\}
$$

the sets of all solutions of the two equations, respectively. Then the following results hold.

(a) The two equations in (4.1) have a common solution if and only if $r\left[\begin{array}{ll}A_{1} & B_{1} \\ A_{2} & B_{2}\end{array}\right]=r\left[\begin{array}{l}A_{1} \\ A_{2}\end{array}\right]$, i.e., $\mathscr{R}\left[\begin{array}{l}B_{1} \\ B_{2}\end{array}\right] \subseteq$ $\mathscr{R}\left[\begin{array}{l}A_{1} \\ A_{2}\end{array}\right]$.

(b) $\mathcal{D}_{1} \subseteq \mathcal{D}_{2}$ if and only if $r\left[\begin{array}{ll}A_{1} & B_{1} \\ A_{2} & B_{2}\end{array}\right]=r\left(A_{1}\right)$, i.e., $\mathscr{R}\left[\begin{array}{l}B_{1} \\ B_{2}\end{array}\right] \subseteq \mathscr{R}\left[\begin{array}{l}A_{1} \\ A_{2}\end{array}\right]$ and $\mathscr{R}\left(A_{2}^{*}\right) \subseteq \mathscr{R}\left(A_{1}^{*}\right)$. 
(c) $\mathcal{D}_{1}=\mathcal{D}_{2}$ if and only if $r\left[\begin{array}{ll}A_{1} & B_{1} \\ A_{2} & B_{2}\end{array}\right]=r\left(A_{1}\right)=r\left(A_{2}\right)$, i.e., $\mathscr{R}\left[\begin{array}{l}B_{1} \\ B_{2}\end{array}\right] \subseteq \mathscr{R}\left[\begin{array}{l}A_{1} \\ A_{2}\end{array}\right]$ and $\mathscr{R}\left(A_{2}^{*}\right)=\mathscr{R}\left(A_{1}^{*}\right)$.

Corollary 4.3. Let $A \in \mathbb{C}^{m \times n}$ and $B \in \mathbb{C}^{m \times p}$ be given, and suppose $A X=B$ is consistent. Also denote

$$
\mathcal{D}_{1}=\{S X \mid A X=B\} \text { and } \mathcal{D}_{2}=\{S X \mid M A X=M B\},
$$

where $M \in \mathbb{C}^{t \times m}$ and $S \in \mathbb{C}^{s \times n}$. Then the following results hold.

(a) $\mathcal{D}_{1} \subseteq \mathcal{D}_{2}$ always holds.

(b) $\mathcal{D}_{1}=\mathcal{D}_{2}$ if and only if $r\left[\begin{array}{c}M A \\ S\end{array}\right]=r\left[\begin{array}{l}A \\ S\end{array}\right]+r(M A)-r(A)$.

Corollary 4.4. Assume that $A X=B$ in (2.5) is consistent, and denote

$$
\mathcal{D}_{1}=\{X \mid A X=B\} \text { and } \mathcal{D}_{2}=\{X \mid M A X=M B\},
$$

where $M \in \mathbb{C}^{s \times m}$. Then, the following results hold.

(a) $\mathcal{D}_{1} \subseteq \mathcal{D}_{2}$ always holds.

(b) $\mathcal{D}_{1}=\mathcal{D}_{2}$ if and only if $r(M A)=r(A)$.

Assume that the matrix equation in (2.5) is consistent, and partition it as

$$
A X=A_{1} X_{1}+A_{2} X_{2}+\cdots+A_{k} X_{k}=B,
$$

where $A_{i} \in \mathbb{C}^{m \times n_{i}}$, with $A=\left[A_{1}, \ldots, A_{k}\right], X_{i} \in \mathbb{C}^{n_{i} \times p}$ are unknown matrices with $X=\left[X_{1}^{\prime}, \ldots, X_{k}^{\prime}\right]^{\prime}$ and $p=p_{1}+\cdots+p_{k}$, and pre-multiplying 4.13) with $P_{Y_{i}}$ yields the following reduced linear matrix equations

$$
P_{Y_{i}} A X=P_{Y_{i}} A_{i} X_{i}=P_{Y_{i}} B, \quad i=1, \ldots, k,
$$

where $Y_{i}=\left[A_{1}, \ldots, A_{i-1}, 0, A_{i+1}, \ldots, A_{k}\right], i=1, \ldots, k$. Then the family of equations in 4.14 are consistent, respectively. In such cases, We denote by

$$
\mathcal{D}_{i}=\left\{X_{i} \mid A_{1} X_{1}+A_{2} X_{2}+\cdots+A_{k} X_{k}=B\right\} \text { and } \mathcal{H}_{i}=\left\{X_{i} \mid E_{Y_{i}} A_{i} X_{i}=E_{Y_{i}} B\right\}, \quad i=1, \ldots, k,
$$

the matrix sets composed by the partial solutions $X_{i}$ of (4.13) and (4.14) respectively; and denote by

$$
\mathcal{D}=\{X \mid A X=B\} \text { and } \mathcal{H}=\left\{\left[X_{1}^{T}, X_{2}^{T}, \ldots, X_{k}^{T}\right]^{T} \mid E_{Y_{i}} A_{i} X_{i}=E_{Y_{i}} B, i=1, \ldots, k\right\} .
$$

In this section, we first discuss the relationships between $\mathcal{D}_{i}$ and $\mathcal{H}_{i}$ in $4.15, i=1, \ldots, k$, as well as the two sets in 4.16.

Theorem 4.5. Assume that the matrix equation in (4.13) is consistent, and let $\mathcal{D}_{i}$ and $\mathcal{H}_{i}$ be as given in (4.15), $i=1, \ldots, k$. Then the following matrix set equalities

$$
\mathcal{D}_{i}=\mathcal{H}_{i}
$$

always hold, $i=1, \ldots, k$.

Proof. Set $S=\left[0 \ldots, I_{n_{i}}, \ldots, 0\right]$ and $M=E_{Y_{i}}$ in 4.11), $i=1, \ldots, k$. Then we obtain by (2.5) and simplifications that

$$
\begin{aligned}
r\left[\begin{array}{c}
E_{Y_{i}} A_{i} \\
S
\end{array}\right]-r\left[\begin{array}{l}
A \\
S
\end{array}\right]-r\left(E_{Y_{i}} A_{i}\right)+r(A) & =r\left[\begin{array}{cc}
A & Y_{i} \\
S & 0
\end{array}\right]-r\left[\begin{array}{c}
A \\
S
\end{array}\right]-r\left[Z_{i}, A_{i}\right]+r(A) \\
& =r\left[\begin{array}{cc}
0 & Y_{i} \\
S & 0
\end{array}\right]-r\left[\begin{array}{c}
Y_{i} \\
S
\end{array}\right]-r(A)+r(A)=0 .
\end{aligned}
$$

Thus 4.17 holds by Corollary 4.3(c).

Theorem 4.6. Assume that the matrix equation in (4.13) is consistent, and let $\mathcal{D}$ and $\mathcal{H}$ be as given in (5.4). Then the following results hold. 
(a) $\mathcal{D} \subseteq \mathcal{H}$ always holds.

(b) The following statements are equivalent:

(i) $\mathcal{D}=\mathcal{H}$.

(ii) $(k-1) r(A)=r\left(Y_{1}\right)+r\left(Y_{2}\right)+\cdots+r\left(Y_{k}\right)$.

(iii) $r(A)=r\left(A_{1}\right)+r\left(A_{2}\right)+\cdots+r\left(A_{k}\right)$.

(iv) $\mathscr{R}\left(Y_{1}\right) \cap \mathscr{R}\left(Y_{2}\right) \cap \cdots \cap \mathscr{R}\left(Y_{k}\right)=\{0\}$.

Proof. By Lemma 2.3, the general solutions of (4.14) are given by

$$
X_{i}=\left(P_{Y_{i}} A_{i}\right)^{\dagger} P_{Y_{i}} B+\left[I_{n_{i}}-\left(P_{Y_{i}} A_{i}\right)^{\dagger}\left(P_{Y_{i}} A_{i}\right)\right] U_{i},
$$

where $U_{i} \in \mathbb{C}^{n_{i} \times p}$ are arbitrary, $i=1, \ldots, k$. Substituting (2.6) and 4.18) into (4.16) gives

$$
\begin{aligned}
& \mathcal{D}=\left\{A^{\dagger} B+Q_{A} U\right\}, \\
& \mathcal{H}=\left\{\left[\begin{array}{c}
\left(P_{Y_{1}} A_{1}\right)^{\dagger} P_{Y_{1}} B \\
\vdots \\
\left(P_{Y_{k}} A_{k}\right)^{\dagger} P_{Y_{k}} B
\end{array}\right]+\left[\begin{array}{ccc}
I_{n_{1}}-P_{Y_{1}} A_{1}\left(P_{Y_{1}} A_{1}\right)^{\dagger} & \ldots & 0 \\
\vdots & \ddots & \vdots \\
0 & \ldots & I_{n_{k}}-P_{Y_{k}} A_{k}\left(P_{Y_{k}} A_{k}\right)^{\dagger}
\end{array}\right]\left[\begin{array}{c}
U_{1} \\
\vdots \\
U_{k}
\end{array}\right]\right\} .
\end{aligned}
$$

Applying Lemma 3.1(b) to 4.19) and 4.20 , we see that $\mathcal{D} \subseteq \mathcal{H}$ if and only if

$$
\begin{aligned}
& r\left[A^{\dagger} B-\left[\begin{array}{c}
\left(P_{Y_{1}} A_{1}\right)^{\dagger} P_{Y_{1}} B \\
\vdots \\
\left(P_{Y_{k}} A_{k}\right)^{\dagger} P_{Y_{k}} B
\end{array}\right], Q_{A},\left[\begin{array}{ccc}
I_{n_{1}}-\left(P_{Y_{1}} A_{1}\right)^{\dagger}\left(P_{Y_{1}} A_{1}\right) & \ldots & 0 \\
\vdots & \ddots & \vdots \\
0 & \ldots & I_{n_{k}}-\left(P_{Y_{k}} A_{k}\right)^{\dagger}\left(P_{Y_{k}} A_{k}\right)
\end{array}\right]\right] \\
& =r\left[\begin{array}{ccc}
I_{n_{1}}-\left(P_{Y_{1}} A_{1}\right)^{\dagger}\left(P_{Y_{1}} A_{1}\right) & \ldots & 0 \\
\vdots & \ddots & \vdots \\
0 & \ldots & I_{n_{k}}-\left(P_{Y_{k}} A_{k}\right)^{\dagger}\left(P_{Y_{k}} A_{k}\right)
\end{array}\right]
\end{aligned}
$$

where by

$$
\begin{aligned}
& r\left[A^{\dagger} B-\left[\begin{array}{c}
\left(P_{Y_{1}} A_{1}\right)^{\dagger} P_{Y_{1}} B \\
\vdots \\
\left(P_{Y_{k}} A_{k}\right)^{\dagger} P_{Y_{k}} B
\end{array}\right], Q_{A},\left[\begin{array}{ccc}
I_{n_{1}}-\left(P_{Y_{1}} A_{1}\right)^{\dagger}\left(P_{Y_{1}} A_{1}\right) & \ldots & 0 \\
\vdots & \ddots & \vdots \\
0 & \ldots & I_{n_{k}}-\left(P_{Y_{k}} A_{k}\right)^{\dagger}\left(P_{Y_{k}} A_{k}\right)
\end{array}\right]\right]
\end{aligned}
$$

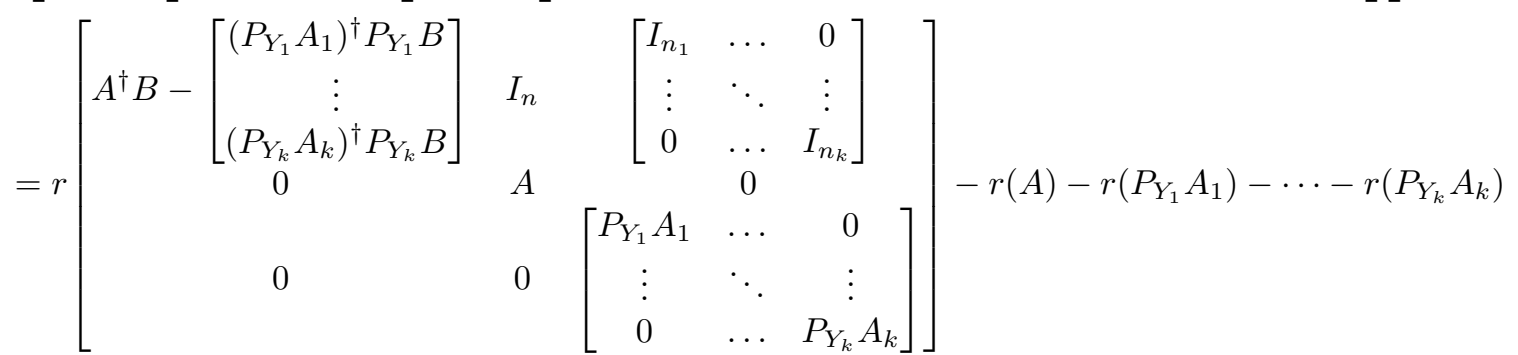

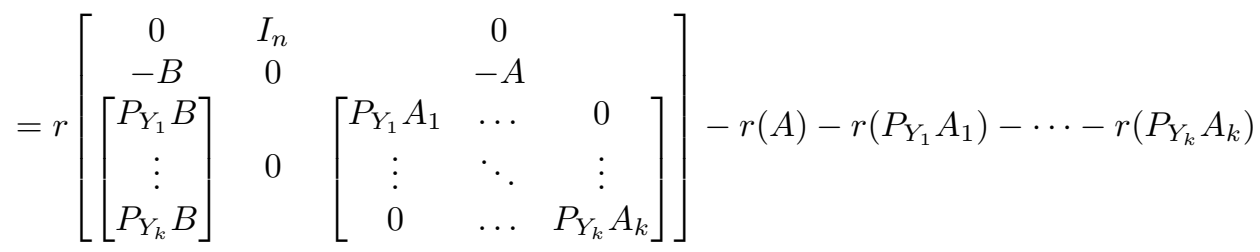

$$
\begin{aligned}
& =n-r\left(P_{Y_{1}} A_{1}\right)-\cdots-r\left(P_{Y_{k}} A_{k}\right) \text {, }
\end{aligned}
$$

and

$$
r\left[\begin{array}{ccc}
I_{n_{1}}-\left(P_{Y_{1}} A_{1}\right)^{\dagger}\left(P_{Y_{1}} A_{1}\right) & \ldots & 0 \\
\vdots & \ddots & \vdots \\
0 & \ldots & I_{n_{k}}-\left(P_{Y_{k}} A_{k}\right)^{\dagger}\left(P_{Y_{k}} A_{k}\right)
\end{array}\right]=n-r\left(P_{Y_{1}} A_{1}\right)-\cdots-r\left(P_{Y_{k}} A_{k}\right) .
$$


Both 4.22 and (4.23) mean that 4.21) is an identity, thus establishing (a).

Substituting (4.18) into (4.13) gives

$$
\begin{aligned}
& {\left[A_{1}-A_{1}\left(P_{Y_{1}} A_{1}\right)^{\dagger}\left(P_{Y_{1}} A_{1}\right)\right] U_{1}+\cdots+\left[A_{k}-A_{k}\left(P_{Y_{k}} A_{k}\right)^{\dagger}\left(P_{Y_{k}} A_{k}\right)\right] U_{k}} \\
& =B-A_{1}\left(P_{Y_{1}} A_{1}\right)^{\dagger} P_{Y_{1}} B+\cdots+A_{k}\left(P_{Y_{k}} A_{k}\right)^{\dagger} P_{Y_{k}} B .
\end{aligned}
$$

It is obvious that $\mathcal{D} \supseteq \mathcal{H}$ holds if and only if the matrix equation in 4.24 holds for all $U_{1}, \ldots, U_{k}$, which by Lemma 2.3 is equivalent to

$$
\begin{aligned}
& {\left[B-A_{1}\left(P_{Y_{1}} A_{1}\right)^{\dagger} P_{Y_{1}} B+\cdots+A_{k}\left(P_{Y_{k}} A_{k}\right)^{\dagger} P_{Y_{k}} B, A_{1}-A_{1}\left(P_{Y_{1}} A_{1}\right)^{\dagger}\left(P_{Y_{1}} A_{1}\right),\right.} \\
& \left.\quad \ldots, A_{k}-A_{k}\left(P_{Y_{k}} A_{k}\right)^{\dagger}\left(P_{Y_{k}} A_{k}\right)\right]=0,
\end{aligned}
$$

where by $(2.5)$,

$$
\begin{aligned}
& r\left[B-A_{1}\left(P_{Y_{1}} A_{1}\right)^{\dagger} P_{Y_{1}} B-\cdots-A_{k}\left(P_{Y_{k}} A_{k}\right)^{\dagger} P_{Y_{k}} B, A_{1}-A_{1}\left(P_{Y_{1}} A_{1}\right)^{\dagger}\left(P_{Y_{1}} A_{1}\right),\right. \\
& \left.\cdots, A_{k}-A_{k}\left(P_{Y_{k}} A_{k}\right)^{\dagger}\left(P_{Y_{k}} A_{k}\right)\right] \\
& =r\left[\begin{array}{cccc}
B-A_{1}\left(P_{Y_{1}} A_{1}\right)^{\dagger} P_{Y_{1}} B-\cdots-A_{k}\left(P_{Y_{k}} A_{k}\right)^{\dagger} P_{Y_{k}} B & A_{1} & \ldots & A_{k} \\
0 & P_{Y_{1}} A_{1} & \ldots & 0 \\
\vdots & \vdots & \ddots & \vdots \\
0 & 0 & \ldots & P_{Y_{k}} A_{k}
\end{array}\right] \\
& -r\left(P_{Y_{1}} A_{1}\right)-\cdots-r\left(P_{Y_{k}} A_{k}\right) \\
& =r\left[\begin{array}{cccc}
B & A_{1} & \ldots & A_{k} \\
P_{Y_{1}} B & P_{Y_{1}} A_{1} & \ldots & 0 \\
\vdots & \vdots & \ddots & \vdots \\
P_{Y_{k}} B & 0 & \ldots & P_{Y_{k}} A_{k}
\end{array}\right]-r\left(P_{Y_{1}} A_{1}\right)-\cdots-r\left(P_{Y_{k}} A_{k}\right) \\
& =r\left[\begin{array}{ccccccc}
B & A_{1} & \ldots & A_{k} & 0 & \ldots & 0 \\
B & A_{1} & \ldots & 0 & Y_{1} & \ldots & 0 \\
\vdots & \vdots & \ddots & \vdots & \vdots & \ddots & \vdots \\
B & 0 & \ldots & A_{k} & 0 & \ldots & Y_{k}
\end{array}\right]-k r(A)=r\left[\begin{array}{ccccc}
B & A & 0 & \ldots & 0 \\
B & A & Y_{1} & \ldots & 0 \\
\vdots & \vdots & \vdots & \ddots & \vdots \\
B & A & 0 & \ldots & Y_{k}
\end{array}\right]-k r(A) \\
& =r\left[\begin{array}{ccccc}
0 & A & 0 & \ldots & 0 \\
0 & 0 & Y_{1} & \ldots & 0 \\
\vdots & \vdots & \vdots & \ddots & \vdots \\
0 & 0 & 0 & \ldots & Y_{k}
\end{array}\right]-k r(A)=r\left(Y_{1}\right)+\cdots+r\left(Y_{k}\right)-(k-1) r(A) .
\end{aligned}
$$

Thus 4.25 is equivalent to $(k-1) r(A)=r\left(Y_{1}\right)+\cdots+r\left(Y_{k}\right)$. Combining this facts with (a) leads to the equivalence of (i) and (ii) in (b). The equivalence of (ii), (iii), and (iv) in (b) follows from Lemma 2.2 .

\section{Relationships among solutions of $A_{1} X_{1} B_{1}+A_{2} X_{2} B_{2}=C$ and its four reduced equations}

Eq. (2.14) is well known in matrix theory and applications, which solvability condition and general solution were precisely established using the ranks, ranges, and generalized inverses of the given matrices in the equation; see e.g., $[2,14,22,23,28$ and the relevant literature quoted there.

It is easy to see that we can construct from (2.14) some small or transformed linear matrix equations. For instance, pre- and post-multiplying (2.14) with $P_{A_{i}}$ and $Q_{B_{i}}$ respectively yield the following four reduced matrix equations

$$
\begin{aligned}
& P_{A_{2}}\left(A_{1} X_{1} B_{1}+A_{2} X_{2} B_{2}\right)=P_{A_{2}} A_{1} X_{1} B_{1}=P_{A_{2}} C, \\
& P_{A_{1}}\left(A_{1} X_{1} B_{1}+A_{2} X_{2} B_{2}\right)=P_{A_{1}} A_{2} X_{2} B_{2}=P_{A_{1}} C, \\
& \left(A_{1} X_{1} B_{1}+A_{2} X_{2} B_{2}\right) Q_{B_{2}}=A_{1} X_{1} B_{1} Q_{B_{2}}=C Q_{B_{2}}, \\
& \left(A_{1} X_{1} B_{1}+A_{2} X_{2} B_{2}\right) Q_{B_{1}}=A_{2} X_{2} B_{2} Q_{B_{1}}=C Q_{B_{1}},
\end{aligned}
$$

respectively. Each of (5.1)-(5.4) is consistent as well, if the matrix equation in (2.14) is consistent. Concerning the relationships among the solutions of (2.14) and (5.1)-(5.4), we have the following results. 
Theorem 5.1. Assume that the matrix equation in (2.14) is consistent, and denote by

$$
\begin{aligned}
& \mathcal{D}=\left\{\left(X_{1}, X_{2}\right) \mid A_{1} X_{1} B_{1}+A_{2} X_{2} B_{2}=C\right\}, \\
& \mathcal{H}_{1}=\left\{\left(X_{1}, X_{2}\right) \mid P_{A_{2}} A_{1} X_{1} B_{1}=P_{A_{2}} C \text { and } P_{A_{1}} A_{2} X_{2} B_{2}=P_{A_{1}} C\right\}, \\
& \mathcal{H}_{2}=\left\{\left(X_{1}, X_{2}\right) \mid P_{A_{2}} A_{1} X_{1} B_{1}=P_{A_{2}} C \text { and } A_{2} X_{2} B_{2} Q_{B_{1}}=C Q_{B_{1}}\right\}, \\
& \mathcal{H}_{3}=\left\{\left(X_{1}, X_{2}\right) \mid A_{1} X_{1} B_{1} Q_{B_{2}}=C Q_{B_{2}} \text { and } P_{A_{1}} A_{2} X_{2} B_{2}=P_{A_{1}} C\right\} \\
& \mathcal{H}_{4}=\left\{\left(X_{1}, X_{2}\right) \mid A_{1} X_{1} B_{1} Q_{B_{2}}=C Q_{B_{2}} \text { and } A_{2} X_{2} B_{2} Q_{B_{1}}=C Q_{B_{1}}\right\},
\end{aligned}
$$

the collections of all pairs of solutions of (2.14) and (5.1)-(5.4), respectively. Then the following results hold.

(a) $\mathcal{D} \subseteq \mathcal{H}_{i}$ always hold, $i=1,2,3,4$.

(b) $\mathcal{D}=\mathcal{H}_{1}$ if and only if $\mathscr{R}\left(A_{1}\right) \cap \mathscr{R}\left(A_{2}\right)=\{0\}$ or $\left[B_{1}^{*}, B_{2}^{*}\right]=0$.

(c) $\mathcal{D}=\mathcal{H}_{2}$ if and only if $A_{2}=0$, or $B_{1}=0$, or $\mathscr{R}\left(A_{1}\right) \cap \mathscr{R}\left(A_{2}\right)=\{0\}$ and $\mathscr{R}\left(B_{1}^{*}\right) \cap \mathscr{R}\left(B_{2}^{*}\right)=\{0\}$.

(d) $\mathcal{D}=\mathcal{H}_{3}$ if and only if $A_{1}=0$, or $B_{2}=0$, or $\mathscr{R}\left(A_{1}\right) \cap \mathscr{R}\left(A_{2}\right)=\{0\}$ and $\mathscr{R}\left(B_{1}^{*}\right) \cap \mathscr{R}\left(B_{2}^{*}\right)=\{0\}$.

(e) $\mathcal{D}=\mathcal{H}_{4}$ if and only if $\left[A_{1}, A_{2}\right]=0$ or $\mathscr{R}\left(B_{1}^{*}\right) \cap \mathscr{R}\left(B_{2}^{*}\right)=\{0\}$.

Proof. Result (a) follows directly from (5.1)-(5.4). By Lemma 2.4, the general solutions of (5.1)-(5.4) are given by

$$
\begin{aligned}
& X_{1}=\left(P_{A_{2}} A_{1}\right)^{\dagger} P_{A_{2}} C B_{1}^{\dagger}+\left[I_{p_{1}}-\left(P_{A_{2}} A_{1}\right)^{\dagger}\left(P_{A_{2}} A_{1}\right)\right] U_{1}+V_{1}\left(I_{q_{1}}-B_{1} B_{1}^{\dagger}\right), \\
& X_{2}=\left(P_{A_{1}} A_{2}\right)^{\dagger} P_{A_{1}} C B_{2}^{\dagger}+\left[I_{p_{2}}-\left(P_{A_{1}} A_{2}\right)^{\dagger}\left(P_{A_{1}} A_{2}\right)\right] U_{2}+V_{2}\left(I_{q_{2}}-B_{2} B_{2}^{\dagger}\right), \\
& X_{1}=A_{1}^{\dagger} C Q_{B_{2}}\left(B_{1} Q_{B_{2}}\right)^{\dagger}+\left(I_{p_{1}}-A_{1}^{\dagger} A_{1}\right) U_{3}+V_{3}\left[I_{q_{1}}-\left(B_{1} Q_{B_{2}}\right)\left(B_{1} Q_{B_{2}}\right)^{\dagger}\right], \\
& X_{2}=A_{2}^{\dagger} C Q_{B_{1}}\left(B_{2} Q_{B_{1}}\right)^{\dagger}+\left(I_{p_{2}}-A_{2}^{\dagger} A_{2}\right) U_{4}+V_{4}\left[I_{q_{2}}-\left(B_{2} Q_{B_{1}}\right)\left(B_{2} Q_{B_{1}}\right)^{\dagger}\right],
\end{aligned}
$$

respectively, where $U_{i}$ and $V_{i}$ are arbitrary matrices, $i=1,2,3,4$. Substituting (5.10)-(5.11) into (2.14) gives the following matrix equation

$$
\begin{aligned}
& A_{1}\left[I_{p_{1}}-\left(P_{A_{2}} A_{1}\right)^{\dagger}\left(P_{A_{2}} A_{1}\right)\right] U_{1} B_{1}+A_{2}\left[I_{p_{2}}-\left(P_{A_{1}} A_{2}\right)^{\dagger}\left(P_{A_{1}} A_{2}\right)\right] U_{2} B_{2} \\
& =C-A_{1}\left(P_{A_{2}} A_{1}\right)^{\dagger} P_{A_{2}} C-A_{2}\left(P_{A_{1}} A_{2}\right)^{\dagger} P_{A_{1}} C, \\
& A_{1}\left[I_{p_{1}}-\left(P_{A_{2}} A_{1}\right)^{\dagger}\left(P_{A_{2}} A_{1}\right)\right] U_{1} B_{1}+A_{2} V_{4}\left[I_{q_{2}}-\left(B_{2} Q_{B_{1}}\right)\left(B_{2} Q_{B_{1}}\right)^{\dagger}\right] B_{2} \\
& =C-A_{1}\left(P_{A_{2}} A_{1}\right)^{\dagger} P_{A_{2}} C-C Q_{B_{1}}\left(B_{2} Q_{B_{1}}\right)^{\dagger} B_{2}, \\
& A_{1} V_{3}\left[I_{q_{1}}-\left(B_{1} Q_{B_{2}}\right)\left(B_{1} Q_{B_{2}}\right)^{\dagger}\right] B_{1}+A_{2}\left[I_{p_{2}}-\left(P_{A_{1}} A_{2}\right)^{\dagger}\left(P_{A_{1}} A_{2}\right)\right] U_{2} B_{2} \\
& =C-C Q_{B_{2}}\left(B_{1} Q_{B_{2}}\right)^{\dagger} B_{1}-A_{2}\left(P_{A_{1}} A_{2}\right)^{\dagger} P_{A_{1}} C, \\
& A_{1} V_{3}\left[I_{q_{1}}-\left(B_{1} Q_{B_{2}}\right)\left(B_{1} Q_{B_{2}}\right)^{\dagger}\right] B_{1}+A_{2} V_{4}\left[I_{q_{2}}-\left(B_{2} Q_{B_{1}}\right)\left(B_{2} Q_{B_{1}}\right)^{\dagger}\right] B_{2} \\
& =C-C Q_{B_{2}}\left(B_{1} Q_{B_{2}}\right)^{\dagger} B_{2}-C Q_{B_{1}}\left(B_{2} Q_{B_{1}}\right)^{\dagger} B_{2},
\end{aligned}
$$

respectively. By Lemma 2.5(b), 5.14 holds for all $U_{1}$ and $U_{2}$ if and only if one of the following four equalities

$$
\begin{aligned}
& {\left[A_{1}\left[I_{p_{1}}-\left(P_{A_{2}} A_{1}\right)^{\dagger}\left(P_{A_{2}} A_{1}\right)\right], A_{2}\left[I_{p_{2}}-\left(P_{A_{1}} A_{2}\right)^{\dagger}\left(P_{A_{1}} A_{2}\right)\right],\right.} \\
& \left.C-A_{1}\left(P_{A_{2}} A_{1}\right)^{\dagger} P_{A_{2}} C-A_{2}\left(P_{A_{1}} A_{2}\right)^{\dagger} P_{A_{1}} C\right]=0, \\
& {\left[\begin{array}{cc}
C-A_{1}\left(P_{A_{2}} A_{1}\right)^{\dagger} P_{A_{2}} C-A_{2}\left(P_{A_{1}} A_{2}\right)^{\dagger} P_{A_{1}} C & A_{1}\left[I_{p_{1}}-\left(P_{A_{2}} A_{1}\right)^{\dagger}\left(P_{A_{2}} A_{1}\right)\right] \\
B_{2} & 0
\end{array}\right]=0,} \\
& {\left[\begin{array}{cc}
C-A_{1}\left(P_{A_{2}} A_{1}\right)^{\dagger} P_{A_{2}} C-A_{2}\left(P_{A_{1}} A_{2}\right)^{\dagger} P_{A_{1}} C & A_{2}\left[I_{p_{2}}-\left(P_{A_{1}} A_{2}\right)^{\dagger}\left(P_{A_{1}} A_{2}\right)\right] \\
B_{1} & 0
\end{array}\right]=0,} \\
& {\left[\begin{array}{c}
C-A_{1}\left(P_{A_{2}} A_{1}\right)^{\dagger} P_{A_{2}} C-A_{2}\left(P_{A_{1}} A_{2}\right)^{\dagger} P_{A_{1}} C \\
B_{1} \\
B_{2}
\end{array}\right]=0 .}
\end{aligned}
$$


It is easy to verify that the ranks of the left-hand sides of $5.18-5.21$ are given by

$$
\begin{aligned}
& r\left[A_{1}\left[I_{p_{1}}-\left(P_{A_{2}} A_{1}\right)^{\dagger}\left(P_{A_{2}} A_{1}\right)\right], A_{2}\left[I_{p_{2}}-\left(P_{A_{1}} A_{2}\right)^{\dagger}\left(P_{A_{1}} A_{2}\right)\right], C-A_{1}\left(P_{A_{2}} A_{1}\right)^{\dagger} P_{A_{2}} C-A_{2}\left(P_{A_{1}} A_{2}\right)^{\dagger} P_{A_{1}} C\right] \\
& =r\left(A_{1}\right)+r\left(A_{2}\right)-r\left[A_{1}, A_{2}\right], \\
& r\left[\begin{array}{cc}
C-A_{1}\left(P_{A_{2}} A_{1}\right)^{\dagger} P_{A_{2}} C-A_{2}\left(P_{A_{1}} A_{2}\right)^{\dagger} P_{A_{1}} C & A_{1}\left[I_{p_{1}}-\left(P_{A_{2}} A_{1}\right)^{\dagger}\left(P_{A_{2}} A_{1}\right)\right] \\
B_{2} & 0
\end{array}\right] \\
& =r\left(A_{1}\right)+r\left(A_{2}\right)-\left[A_{1}, A_{2}\right]+r\left(B_{2}\right), \\
& r\left[\begin{array}{cc}
C-A_{1}\left(P_{A_{2}} A_{1}\right)^{\dagger} P_{A_{2}} C-A_{2}\left(P_{A_{1}} A_{2}\right)^{\dagger} P_{A_{1}} C & A_{2}\left[I_{p_{2}}-\left(P_{A_{1}} A_{2}\right)^{\dagger}\left(P_{A_{1}} A_{2}\right)\right] \\
B_{1}
\end{array}\right] \\
& =r\left(A_{1}\right)+r\left(A_{2}\right)-\left[\begin{array}{c}
\left.A_{1}, A_{2}\right]+r\left(B_{1}\right), \\
A_{1}
\end{array}\right]=r\left[\begin{array}{l}
B_{1} \\
B_{2}
\end{array}\right] .
\end{aligned}
$$

Combining (5.18)-(5.21) with $5.22-(5.25)$ leads to the equivalence in (b).

By Lemma 2.5(b), 5.15 holds for all $U_{1}$ and $V_{4}$ if and only if one of the following four equalities

$$
\begin{aligned}
& {\left[C-A_{1}\left(P_{A_{2}} A_{1}\right)^{\dagger} P_{A_{2}} C-A_{2}\left(P_{A_{1}} A_{2}\right)^{\dagger} P_{A_{1}} C, A_{1}\left[I_{p_{1}}-\left(P_{A_{2}} A_{1}\right)^{\dagger}\left(P_{A_{2}} A_{1}\right)\right], A_{2}\right]=0,} \\
& {\left[\begin{array}{cc}
C-A_{1}\left(P_{A_{2}} A_{1}\right)^{\dagger} P_{A_{2}} C B_{1}^{\dagger} B_{1}-A_{2} A_{2}^{\dagger} C Q_{B_{1}}\left(B_{2} Q_{B_{1}}\right)^{\dagger} B_{2} & A_{1}\left[I_{p_{1}}-\left(P_{A_{2}} A_{1}\right)^{\dagger}\left(P_{A_{2}} A_{1}\right)\right] \\
{\left[I_{q_{2}}-\left(B_{2} Q_{B_{1}}\right)\left(B_{2} Q_{B_{1}}\right)^{\dagger}\right] B_{2}} & 0
\end{array}\right]=0,} \\
& {\left[\begin{array}{cc}
C-A_{1}\left(P_{A_{2}} A_{1}\right)^{\dagger} P_{A_{2}} C B_{1}^{\dagger} B_{1}-A_{2} A_{2}^{\dagger} C Q_{B_{1}}\left(B_{2} Q_{B_{1}}\right)^{\dagger} B_{2} & A_{1} \\
B_{2} & 0
\end{array}\right]=0,} \\
& {\left[\begin{array}{c}
C-A_{1}\left(P_{A_{2}} A_{1}\right)^{\dagger} P_{A_{2}} C B_{1}^{\dagger} B_{1}-A_{2} A_{2}^{\dagger} C Q_{B_{1}}\left(B_{2} Q_{B_{1}}\right)^{\dagger} B_{2} \\
B_{1} \\
{\left[I_{q_{2}}-\left(B_{2} Q_{B_{1}}\right)\left(B_{2} Q_{B_{1}}\right)^{\dagger}\right] B_{2}}
\end{array}\right]=0 .}
\end{aligned}
$$

It is easy to verify that the ranks of the left-hand sides of $(5.26)-(5.29)$ are given by

$$
\begin{aligned}
& r\left[C-A_{1}\left(P_{A_{2}} A_{1}\right)^{\dagger} P_{A_{2}} C-A_{2}\left(P_{A_{1}} A_{2}\right)^{\dagger} P_{A_{1}} C, A_{1}\left[I_{p_{1}}-\left(P_{A_{2}} A_{1}\right)^{\dagger}\left(P_{A_{2}} A_{1}\right)\right], A_{2}\right]=r\left(A_{2}\right), \\
& r\left[\begin{array}{cc}
C-A_{1}\left(P_{A_{2}} A_{1}\right)^{\dagger} P_{A_{2}} C B_{1}^{\dagger} B_{1}-A_{2} A_{2}^{\dagger} C Q_{B_{1}}\left(B_{2} Q_{B_{1}}\right)^{\dagger} B_{2} & A_{1}\left[I_{p_{1}}-\left(P_{A_{2}} A_{1}\right)^{\dagger}\left(P_{A_{2}} A_{1}\right)\right] \\
{\left[I_{q_{2}}-\left(B_{2} Q_{B_{1}}\right)\left(B_{2} Q_{B_{1}}\right)^{\dagger}\right] B_{2}} & 0
\end{array}\right] \\
& =r\left(A_{1}\right)+r\left(A_{2}\right)+r\left(B_{1}\right)+r\left(B_{2}\right)-r\left[A_{1}, A_{2}\right]-r\left[\begin{array}{c}
B_{1} \\
B_{2}
\end{array}\right], \\
& r\left[\begin{array}{cc}
C-A_{1}\left(P_{A_{2}} A_{1}\right)^{\dagger} P_{A_{2}} C B_{1}^{\dagger} B_{1}-A_{2} A_{2}^{\dagger} C Q_{B_{1}}\left(B_{2} Q_{B_{1}}\right)^{\dagger} B_{2} & A_{1} \\
B_{2} & 0
\end{array}\right]=r\left(A_{1}\right)+r\left(B_{2}\right), \\
& r\left[\begin{array}{c}
C-A_{1}\left(P_{A_{2}} A_{1}\right)^{\dagger} P_{A_{2}} C B_{1}^{\dagger} B_{1}-A_{2} A_{2}^{\dagger} C Q_{B_{1}}\left(B_{2} Q_{B_{1}}\right)^{\dagger} B_{2} \\
B_{1}
\end{array}\right]=r\left(B_{1}\right) . \\
& {\left[I_{q_{2}}-\left(B_{2} Q_{B_{1}}\right)\left(B_{2} Q_{B_{1}}\right)^{\dagger}\right] B_{2}}
\end{aligned}
$$

Combining (5.26)-(5.29) with (5.30)-5.33 leads to the equivalence in (c). Results (d) and (e) can be established by a similar approach.

Theorem 5.2. Assume that the matrix equation in (2.14) is consistent, and let

$$
\begin{aligned}
& \mathcal{D}_{1}=\left\{X_{1} \mid A_{1} X_{1} B_{1}+A_{2} X_{2} B_{2}=C\right\}, \\
& \mathcal{D}_{2}=\left\{X_{2} \mid A_{1} X_{1} B_{1}+A_{2} X_{2} B_{2}=C\right\}, \\
& \mathcal{H}_{1}=\left\{X_{1} \mid A_{1} X_{1} B_{1}-A_{2} A_{2}^{\dagger} A_{1} X_{1} B_{1} B_{2}^{\dagger} B_{2}=C-A_{2} A_{2}^{\dagger} C B_{2}^{\dagger} B_{2}\right\}, \\
& \mathcal{H}_{2}=\left\{X_{2} \mid A_{2} X_{2} B_{2}-A_{1} A_{1}^{\dagger} A_{2} X_{2} B_{2} B_{1}^{\dagger} B_{1}=C-A_{1} A_{1}^{\dagger} C B_{1}^{\dagger} B_{1}\right\}, \\
& \mathcal{D}=\left\{\left(X_{1}, X_{2}\right) \mid A_{1} X_{1} B_{1}+A_{2} X_{2} B_{2}=C\right\}, \\
& \mathcal{H}=\left\{\left(X_{1}, X_{2}\right) \mid X_{1} \in \mathcal{H}_{1} \text { and } X_{2} \in \mathcal{H}_{2}\right\} .
\end{aligned}
$$

Then the following results hold. 
(a) The matrix set equalities $\mathcal{D}_{i}=\mathcal{H}_{i}$ always hold, $i=1,2$.

(b) $\mathcal{D} \subseteq \mathcal{H}$ always holds.

(c) $\mathcal{D}=\mathcal{H}$ if and only if $\mathscr{R}\left(B_{1}^{\top} \otimes A_{1}\right) \cap \mathscr{R}\left(B_{2}^{\top} \otimes A_{2}\right)=\{0\}$.

Proof. By the vec operation of matrix, (2.14) can equivalently be expressed as

$$
\left(B_{1}^{\top} \otimes A_{1}\right) \vec{X}_{1}+\left(B_{2}^{\top} \otimes A_{2}\right) \vec{X}_{2}=\vec{C},
$$

which is a special case of (4.13). Pre-multiplying [5.40 with $P_{B_{i}^{\top} \otimes A_{i}}$ yields the following reduced linear matrix equation equations

$$
P_{B_{2}^{\top} \otimes A_{2}}\left(B_{1}^{\top} \otimes A_{1}\right) \vec{X}_{1}=P_{B_{2}^{\top} \otimes A_{2}} \vec{C}, \quad P_{B_{1}^{\top} \otimes A_{1}}\left(B_{2}^{\top} \otimes A_{2}\right) \vec{X}_{2}=P_{B_{2}^{\top} \otimes A_{2}} \vec{C}
$$

Now denote

$$
\begin{aligned}
& \widehat{\mathcal{D}}_{i}=\left\{\vec{X}_{i} \mid\left(B_{1}^{\top} \otimes A_{1}\right) \vec{X}_{1}+\left(B_{2}^{\top} \otimes A_{2}\right) \vec{X}_{2}=\vec{C}\right\}, \quad i=1,2, \\
& \widehat{\mathcal{H}}_{1}=\left\{\vec{X}_{1} \mid P_{B_{2}^{\top} \otimes A_{2}}\left(B_{1}^{\top} \otimes A_{1}\right) \vec{X}_{1}=P_{B_{2}^{\top} \otimes A_{2}} \vec{C}\right\}, \\
& \widehat{\mathcal{H}}_{2}=\left\{\vec{X}_{2} \mid P_{B_{1}^{\top} \otimes A_{1}}\left(B_{2}^{\top} \otimes A_{2}\right) \vec{X}_{2}=P_{B_{1}^{\top} \otimes A_{1}} \vec{C}\right\} .
\end{aligned}
$$

Then by Corollary 4.5

$$
\widehat{\mathcal{D}}_{i}=\widehat{\mathcal{H}}_{i}, \quad i=1,2
$$

always hold. On the other hand, it is easy to verify that

$$
P_{B_{i}^{\top} \otimes A_{i}}=I_{m n}-\left(B_{i}^{\top} \otimes A_{i}\right)\left(B_{i}^{\top} \otimes A_{i}\right)^{\dagger}=I_{m n}-\left(B_{i}^{\top} \otimes A_{i}\right)\left[\left(B_{i}^{\top}\right)^{\dagger} \otimes A_{i}^{\dagger}\right]=I_{m n}-\left(B_{i}^{\dagger} B_{i}\right)^{\top} \otimes A_{i} A_{i}^{\dagger}, \quad i=1,2,
$$

and

$$
\begin{aligned}
& P_{B_{2}^{\top} \otimes A_{2}}\left(B_{1}^{\top} \otimes A_{1}\right)=B_{1}^{\top} \otimes A_{1}-\left[\left(B_{2}^{\dagger} B_{2}\right)^{\top} \otimes A_{2} A_{2}^{\dagger}\right]\left(B_{1}^{\top} \otimes A_{1}\right)=B_{1}^{\top} \otimes A_{1}-\left(B_{1} B_{2}^{\dagger} B_{2}\right)^{\top} \otimes A_{2} A_{2}^{\dagger} A_{1}, \\
& P_{B_{1}^{\top} \otimes A_{1}}\left(B_{2}^{\top} \otimes A_{2}\right)=B_{2}^{\top} \otimes A_{2}-\left(B_{1}^{\dagger} B_{1}\right)^{\top} \otimes A_{1} A_{1}^{\dagger}\left(B_{2}^{\top} \otimes A_{2}\right)=B_{2}^{\top} \otimes A_{2}-\left(B_{2} B_{1}^{\dagger} B_{1}\right)^{\top} \otimes A_{1} A_{1}^{\dagger} A_{2} .
\end{aligned}
$$

Thus the two equations in 5.41 by the vectorization operation of matrix are equivalent to

$$
\begin{aligned}
& A_{1} X_{1} B_{1}-A_{2} A_{2}^{\dagger} A_{1} X_{1} B_{1} B_{2}^{\dagger} B_{2}=C-A_{2} A_{2}^{\dagger} C B_{2}^{\dagger} B_{2}, \\
& A_{2} X_{2} B_{2}-A_{1} A_{1}^{\dagger} A_{2} X_{2} B_{2} B_{1}^{\dagger} B_{1}=C-A_{1} A_{1}^{\dagger} C B_{1}^{\dagger} B_{1},
\end{aligned}
$$

respectively. Thus the two set equalities in 5.45 are equivalent to the set equalities in (a). Results (b) and (c) follow from applying Theorem 4.6 to $(5.40)$.

In addition to the LMVF in (1.3), there are many types of multilinear and nonlinear matrix-valued functions that occur in matrix theory and applications, such as,

$$
\begin{aligned}
f\left(X_{1}, \ldots, X_{k}\right) & =\left(A_{1}+B_{1} X_{1} C_{1}\right)\left(A_{2}+B_{2} X_{2} C_{2}\right) \cdots\left(A_{k}+B_{k} X_{k} C_{k}\right), \\
g\left(X_{1}, Y_{1}, \ldots, X_{k}, Y_{k}\right) & =\left(A_{1}+B_{1} X_{1} C_{1}+D_{1} Y_{1} E_{1}\right) \cdots\left(A_{k}+B_{k} X_{k} C_{k}+D_{k} Y_{k} E_{k}\right),
\end{aligned}
$$

etc. In these cases, it would be of interest but are also challenging to investigate the connections between a pair of such matrix-valued functions under various specified assumptions.

\section{References}

[1] M. Bakonyi, H.J. Woerdeman. Matrix completions, moments, and sums of Hermitian squares. Princeton University Press, Princeton, NJ, 2011.

[2] J.K. Baksalary, R. Kala. The matrix equation $A X B+C Y D=E$. Linear Algebra Appl. 30(1980), $141-147$.

[3] R. Horn, C.R. Johnson. Topics in Matrix Analysis. Cambridge University Press, 1991. 
[4] A. Ben-Israel and T.N.E. Greville. Generalized Inverses: Theory and Applications. 2nd ed., Springer-Verlag, New York, 2003.

[5] S.L. Campbell and C.D. Meyer Jr. Generalized Inverses of Linear Transformations. Corrected reprint of the 1979 original, Dover Publications, Inc., New York, 1991.

[6] T. Damm, H.K. Wimmer. A cancellation property of the Moore-Penrose inverse of triple products. J. Aust. Math. Soc. 86(2009), 33-44.

[7] J. Dancis. Choosing the inertias for completions of certain partially specified matrices. SIAM J. Matrix Anal. Appl. 14(1993), 813-829.

[8] I. Gohberg, M.A. Kaashoek, F.V. Schagen. Partially Specified Matrices and Operators: Classification, Completion, Applications. Birkhäuser Basel, 1995.

[9] R.E. Hartwig. The reverse order law revisited. Linear Algebra Appl. 76(1986), 241-246.

[10] B. Jiang, Y. Tian. Necessary and sufficient conditions for nonlinear matrix identities to always hold. Aequat. Math. 93(2019), 587-600.

[11] C. Jordán, J.R. Torregrosa, A. Urbano. On the jordan form of completions of partial upper triangular matrices. Linear Algebra Appl. 1997, 254(1997), 241-250.

[12] M. Krupnik. Geometric multiplicities of completions of partial triangular matrices. Linear Algebra Appl. 220(1995), 215-227.

[13] G. Marsaglia, G.P.H. Styan. Equalities and inequalities for ranks of matrices. Linear Multilinear Algebra 2(1974), 269-292.

[14] A.B. Özgüler. The matrix equation $A X B+C Y D=E$ over a principal ideal domain. SIAM J. Matrix. Anal. Appl. 12(1991), 581-591.

[15] S.K. Mitra. Common solutions to a pair of linear matrix equations $A_{1} X B_{1}=C_{1}$ and $A_{2} X B_{2}=C_{2}$. Proc. Cambridge Philos. Soc. 74(1973), 213-216.

[16] S.K. Mitra. A pair of simultaneous linear matrix equations and a matrix programming problem Linear Algebra Appl. 131(1990), 97-123.

[17] A. Navarra, P.L. Odell, D.M. Young. A representation of the general common solution to the matrix equations $A_{1} X B_{1}=C_{1}$ and $A_{2} X B_{2}=C_{2}$ with applications. Comput. Math. Appl. 41(2001), 929-935.

[18] C.R. Rao, S.K. Mitra. Generalized Inverse of Matrices and Its Applications. Wiley, New York, 1971.

[19] R. Penrose. A generalized inverse for matrices. Proc. Cambridge Phil. Soc. 51(1955), 406-413.

[20] W.-H. Steeb. Matrix Calculus and Kronecker Product with Applications and C++ Programs. World Scientific Publishing, 1997.

[21] Y. Tian. Solvability of two linear matrix equations. Linear Multilinear Algebra 48(2000), $123-147$.

[22] Y. Tian. Upper and lower bounds for ranks of matrix expressions using generalized inverses. Linear Algebra Appl. $355(2002), 187-214$.

[23] Y. Tian. Ranks and independence of solutions of the matrix equation $A X B+C Y D=M$. Acta Math. Univ. Comenianae 75(2006), 75-84.

[24] Y. Tian. On additive decompositions of solutions of the matrix equation $A X B=C$. Calcolo 47(2010), $193-209$.

[25] Y. Tian. Relations between matrix sets generated from linear matrix expressions and their applications. Comput. Math. Appl. 61(2011), 1493-1501.

[26] Y. Tian. Formulas for calculating the dimensions of the sums and the intersections of a family of linear subspaces with applications. Beitr. Algebra Geom. 60(2019), 471-485.

[27] Y. Tian. Classification analysis to the equalities $A^{(i, \ldots, j)}=B^{(k, \ldots, l)}$ for generalized inverses of two matrices. Linear Multilinear Algebra, 2019, doi:10.1080/03081087.2019.1627279.

[28] G. Xu, M. Wei, D. Zheng. On solution of matrix equation $A X B+C Y D=F$. Linear Algebra Appl. 279(1998), 93-109. 